OPEN ACCESS

Check for updates

For numbered affiliations see

end of the article.

Correspondence to:

EAAklea32@aub.edu.lb

(or @elie_akl on Twitter:

ORCID 0000-0002-3444-8618)

Additional material is published online only. To view please visit

the journal online.

Cite this as: $B M / 2020 ; 370: \mathrm{m} 2898$ http://dx.doi.org/10.1136/bmj.m2898

Accepted: 30 June 2020

\section{Potential impact of missing outcome data on treatment effects in systematic reviews: imputation study}

\author{
Lara A Kahale, ${ }^{1,2}$ Assem M Khamis, ${ }^{3}$ Batoul Diab, ${ }^{1}$ Yaping Chang, ${ }^{4}$ Luciane Cruz Lopes, ${ }^{5}$ \\ Arnav Agarwal, ${ }^{4,6}$ Ling Li, ${ }^{7}$ Reem A Mustafa, ${ }^{4,8}$ Serge Koujanian, ${ }^{9}$ Reem Waziry, ${ }^{10}$ \\ Jason W Busse, ${ }^{4,11,12,13}$ Abeer Dakik, ${ }^{1}$ Holger J Schünemann, ${ }^{4}$ Lotty Hooft, ${ }^{2}$ Rob JPM Scholten, ${ }^{2}$ \\ Gordon H Guyatt, ${ }^{4,14}$ Elie A Akl ${ }^{14}$
}

\section{ABSTRACT}

OBJECTIVE

To assess the risk of bias associated with missing outcome data in systematic reviews.

DESIGN

Imputation study.

SETTING

Systematic reviews.

POPULATION

100 systematic reviews that included a group level meta-analysis with a statistically significant effect on a patient important dichotomous efficacy outcome.

\section{MAIN OUTCOME MEASURES}

Median percentage change in the relative effect estimate when applying each of the following assumption (four commonly discussed but implausible assumptions (best case scenario, none had the event, all had the event, and worst case scenario) and four plausible assumptions for missing data based on the informative missingness odds ratio (IMOR) approach (IMOR 1.5 (least stringent), IMOR 2, IMOR 3, IMOR 5 (most stringent)); percentage of meta-analyses that crossed the threshold of the null effect for each method; and percentage of metaanalyses that qualitatively changed direction of effect for each method. Sensitivity analyses based on the eight different methods of handling missing data were conducted.

RESULTS

100 systematic reviews with 653 randomised controlled trials were included. When applying the implausible but commonly discussed assumptions,

\section{WHAT IS ALREADY KNOWN ON THIS TOPIC}

Missing data on the outcomes of participants in randomised controlled trials might introduce bias in systematic reviews

To assess that risk of bias, the GRADE (grading of recommendations assessment, development, and evaluation) working group recommends challenging the robustness of the meta-analysis effect estimate by conducting sensitivity analyses with different methods of handling missing data

\section{WHAT THIS STUDY ADDS}

Even when applying plausible assumptions to the outcomes of participants with definite missing data, the average change in pooled relative effect estimate is substantive, whereas almost a quarter (22\%) of meta-analyses crossed the threshold of the null effect

Systematic review authors should present the potential impact of missing outcome data on their effect estimates and use this to inform their overall GRADE ratings of risk of bias and their interpretation of the results the median change in the relative effect estimate varied from $0 \%$ to $30.4 \%$. The percentage of metaanalyses crossing the threshold of the null effect varied from $1 \%$ (best case scenario) to $60 \%$ (worst case scenario), and $26 \%$ changed direction with the worst case scenario. When applying the plausible assumptions, the median percentage change in relative effect estimate varied from $1.4 \%$ to $7.0 \%$. The percentage of meta-analyses crossing the threshold of the null effect varied from $6 \%$ (IMOR 1.5) to $22 \%$ (IMOR 5) of meta-analyses, and $2 \%$ changed direction with the most stringent (IMOR 5).

\section{CONCLUSION}

Even when applying plausible assumptions to the outcomes of participants with definite missing data, the average change in pooled relative effect estimate is substantive, and almost a quarter (22\%) of meta-analyses crossed the threshold of the null effect. Systematic review authors should present the potential impact of missing outcome data on their effect estimates and use this to inform their overall GRADE (grading of recommendations assessment, development, and evaluation) ratings of risk of bias and their interpretation of the results.

\section{Introduction}

Despite efforts to reduce the occurrence of missing outcome data in clinical trials, ${ }^{1}$ this is still common. Across six methodological surveys the percentage of randomised controlled trials with missing outcome data ranged from $63 \%$ to $100 \%,{ }^{2-7}$ and the average proportion of participants with missing outcome data among trials reporting missing data ranged from $6 \%$ to $24 \% .^{2-8}$ Among 235 randomised controlled trials with statistically significant results published in leading medical journals, one in three lost statistical significance when making plausible assumptions about the outcomes of participants with missing data. ${ }^{2}$ Another study comparing different approaches to modelling binary outcomes with missing data in an alcohol clinical trial yielded different results with various amount of bias depending on the approach and missing data scenario. ${ }^{9}$

The extent of missing outcome data in randomised controlled trials contributes to the risk of bias of meta-analyses involving those trials. Additional factors that might bias the results of meta-analysis include the methods used by contributing randomised controlled trials to handle missing data and the transparency of reporting missing data (for each arm and follow-up time point). To explore the impact on 
risk of bias, the GRADE (grading of recommendations assessment, development, and evaluation) working group recommends conducting sensitivity analyses using assumptions regarding the outcomes of patients with missing outcome data. ${ }^{10} \mathrm{~A}$ recent empirical comparison of bayesian modelling strategies for missing binary outcome data in network meta-analysis found that using implausible assumptions could negatively affect network meta-analysis estimates. ${ }^{11}$ No methodological study has yet assessed the impact of different assumptions about missing data on the robustness of the pooled relative effect in a large representative sample of published systematic reviews of pairwise comparisons.

One challenge when handling missing data is the lack of clarity in trial reports on whether participants have missing outcome data. ${ }^{12}$ We recently published guidance for authors of systematic reviews on how to identify and classify participants with missing outcome data in the trial reports. ${ }^{13}$ The Cochrane Handbook acknowledges that attempts to deal with missing data in systematic reviews are often hampered by incomplete reporting of missing outcome data by trialists. ${ }^{14}$ A recent methodological survey among 638 randomised controlled trials reported that the median percentage of participants with unclear follow-up status was $9.7 \%$ (interquartile range $4.1-19.9 \%),{ }^{8}$ and that when authors explicitly reported not followingup participants, almost half did not specify how they handled missing data in their analysis.

We assessed risk of bias associated with missing outcome data in systematic reviews with two interventions by quantifying the change in effect estimate when applying different methods of handling missing outcome data; examining how these methods alter crossing the threshold of the null effect of pooled effect estimates; qualitatively changing the direction of effect; and exploring the potential effect on heterogeneity of each of these approaches. ${ }^{4}$

\section{Methods}

Design

This study is part of a larger project examining methodological problems related to missing data in systematic reviews and randomised controlled trials. ${ }^{15}$ Our published protocol includes detailed information on the definitions, eligibility criteria, search strategy, selection process, data abstraction, and data analysis. ${ }^{15}$ In the appendix (supplementary table 1), we present deviations from the protocol and the rationale for these deviations.

We defined missing data as outcome data for trial participants that are not available to authors of systematic reviews (from the published randomised controlled trial reports or personal contact with the trial authors).

In the current study, we collected a random sample of 50 Cochrane and 50 non-Cochrane systematic reviews published in 2012 that reported a group level meta-analysis of a patient important dichotomous efficacy outcome, with a statistically significant effect estimate (the meta-analysis of interest). ${ }^{16}$ We used the term original pooled relative effect to refer to the result of the meta-analysis as reported by the systematic review authors. For the individual trials included in the meta-analyses of interest, ${ }^{8}$ we abstracted detailed information relevant to the statistical analysis and missing data and conducted sensitivity meta-analyses based on nine different methods of handling missing data. Our outcomes were the median change of effect estimates across meta-analyses; the percentage of meta-analyses that crossed the threshold of the null effect with each of these methods; the percentage of meta-analyses that changed direction of effect; and the change in heterogeneity associated with each of the methods.

\section{Identifying participants with missing data}

Since publication of our protocol, we published a guidance for authors of systematic reviews on how to identify and classify participants with missing outcome data in the trial reports depending on how trial authors report on those categories and handle them in their analyses (table 1). ${ }^{13}$ The guidance includes a taxonomy of categories of trial participants who might have missing data, along with a description of those categories (supplementary table 2). The categorisation reflects the wording used in trial reports-that is, the presentation faced by systematic review authors. We used this guidance to judge the outcome data missingness of categories of participants who might have missing data (ie, whether they have definite, potential, or no missing data).

\section{Data abstraction}

Eleven reviewers trained in health research methodology abstracted the data independently and in duplicate. The reviewers met regularly with a core team (EAA, LAK, $\mathrm{BD}$, and $\mathrm{AD}$ ) to discuss progress and challenges and develop solutions. We used a pilot tested standardised data abstraction form hosted in an electronic data capture tool, REDCap. ${ }^{17}$ All reviewers underwent calibration exercises before data abstraction to improve reliability, and a senior investigator served as a third independent reviewer for resolving disagreements.

From each eligible meta-analysis we abstracted the original (published) pooled relative effect-that is, pooled relative effect measure (relative risk or odds ratio) and the associated 95\% confidence interval, the analysis model used (random effects or fixed effect), and the statistical method used for pooling data (eg, Mantel-Haenszel, Peto).

For each study arm in the randomised controlled trials, we abstracted the numbers of participants randomised, events, participants with definite missing data (according to the suggested guidance on identifying participants with missing data ${ }^{13}$ ), and participants with potentially missing data.

\section{Data analysis}

SPSS statistical software, version 21.0 was used to conduct a descriptive analysis of study characteristics 


\begin{tabular}{lc}
\hline $\begin{array}{l}\text { Table } 1 \text { | Judging missingness of outcome data on the basis of reporting and handling of categories of participants who } \\
\text { might have missing data }\end{array}$ & $\begin{array}{l}\text { Judgment of outcome } \\
\text { data missingness }\end{array}$ \\
$\begin{array}{l}\text { Categories of participants } \\
\text { Participants explicitly reported as followed-up, participants who died during the trial, } \\
\text { participants belonging to centres that were excluded }\end{array}$ & Definitely not missing data \\
\hline $\begin{array}{l}\text { Participants explicitly reported as not followed-up, and participants with unclear follow-up status and who } \\
\text { were excluded from the denominator of the analysis (ie, complete case analysis) or were included in the } \\
\text { denominator of the analysis and their outcomes were explicitly stated to be imputed }\end{array}$ & Definite missing data \\
\hline $\begin{array}{l}\text { Participants with unclear follow-up status (eg, included in the denominator of the analysis and their } \\
\text { outcomes were not explicitly stated to be imputed) }\end{array}$ & Potential missing data \\
\hline Participants with definite or potential missing data & Total possible missing data \\
\hline
\end{tabular}

of eligible systematic reviews and the associated randomised controlled trials. ${ }^{18}$ For categorical variables, we report frequencies and percentages, and for continuous variables that were not normally distributed, we use medians and interquartile ranges.

To explore the robustness of pooled effect estimates reported by systematic reviews, we conducted several sensitivity analyses based on nine different methods of handling missing data, using Stata software release $12^{19}$ (see table 2):

- Complete case analysis ${ }^{10} 20$

- Four implausible but commonly discussed assumptions: best case scenario, none of the participants with missing data had the outcome, all participants with missing data had the outcome, and worst case scenario.

- Four plausible assumptions using increasingly stringent values for informative missing odds ratio (IMOR) in the intervention arm. ${ }^{21} 22$ IMOR describes the ratio of odds of the outcome among participants with missing data to the odds of the outcome among observed participants. In other words, to obtain the odds among participants with missing data, the odds is multiplied among the observed participants with a stringent value (ie, 1.5, 2, 3, and 5). We chose an upper limit of 5 as it represents the highest ratio reported in the literature. One study used a community tracker to evaluate the incidence of death among participants in scale-up programmes of antiretroviral treatment in Africa who were lost to follow-up. ${ }^{2324}$ The study found the mortality rate to be five times higher in patients lost to followup compared with patients who were followed up. We did not use an IMOR of 1 because it provides the same effect estimate as the complete case analysis.

Naïve single imputation methods falsely increase precision by treating imputed outcome data as if they were observed, whereas IMORs (or pattern mixture models in general), by imputing risks, increase uncertainty within a trial to deal with the fact that data have been imputed. Imputing events consists of including participants with missing data in the denominator and making assumptions about their outcomes in the numerator. This approach might lead to imputing several events as if they were fully observed, leading to possibly problematic narrower confidence intervals than would be the case. To correct for this, methodologists have developed methods that account for uncertainty associated with imputing missing observations using statistical approaches. ${ }^{21} 2227$ The command "metamiss" in Stata integrates uncertainty within its calculation ${ }^{25} 26$ (see statistical notes in appendix for further details).

We did not consider the nature of outcomes (positive versus negative) during the conduct of the sensitivity analyses best case and worst case scenarios. Instead, we focused in the sensitivity analyses on challenging the effect estimates against the null value when the best case scenario shifts the effect estimate away from the null value of 1 and the worst case scenario shifts the effect estimate towards the null value of 1 (irrespective of whether the outcome is positive or negative).

Our analytical approach was executed in one command (metamiss ${ }^{25}$ ) of Stata release $12^{19}$ for each sensitivity analysis. Firstly, we recalculated each meta-analysis of interest, for all 100 systematic reviews, using each method to deal with missing outcome data to generate different sensitivity analysis pooled effects along with the corresponding 95\% confidence intervals. We used the same relative effect measure (relative risk or odds ratio), the same analysis model (random effects or fixed effect), and the same statistical method (eg, Mantel-Haenszel, Peto) as the original meta-analysis of interest. Secondly, across all included meta-analyses and for each method, we explored the impact of the revised meta-analysis for several outcomes:

\section{Change in relative effect estimate}

To quantify the percentage change in relative effect estimate between the sensitivity analysis pooled effect estimate (assumption) and the sensitivity analysis pooled effect estimate (complete case analysis), we applied the formula (fig 1; see statistical notes in appendix for further details).

We calculated specifically the percentage of metaanalyses with change of relative effect estimate (by direction) between the sensitivity analysis pooled effect estimate (assumption) and the sensitivity analysis pooled effect estimate (complete case analysis) and the median and interquartile range for the change in relative effect estimate (stratified by direction of change).

This relative change could be an increase or a reduction in effect. For example, a relative increase 


\begin{tabular}{|c|c|c|c|c|}
\hline \multirow{2}{*}{$\begin{array}{l}\text { Method of handling } \\
\text { missing data }\end{array}$} & \multicolumn{4}{|c|}{ Handling participants with missing data in the numerator and denominator } \\
\hline & Intervention arm & & Control arm & \\
\hline \multicolumn{5}{|l|}{$\begin{array}{l}\text { Implausible but commonly } \\
\text { discussed assumptions: }\end{array}$} \\
\hline Best case scenario* & $\begin{array}{l}\text { Assumed that all had a } \\
\text { favourable outcome }\end{array}$ & Denominator included & $\begin{array}{l}\text { Assumed that all had an } \\
\text { unfavourable outcome }\end{array}$ & Denominator included \\
\hline $\begin{array}{l}\text { None of the participants with } \\
\text { missing data had the outcome }\end{array}$ & $\begin{array}{l}\text { Assumed that none } \\
\text { had the outcome }\end{array}$ & Denominator included & $\begin{array}{l}\text { Assumed that none } \\
\text { had the outcome }\end{array}$ & Denominator included \\
\hline $\begin{array}{l}\text { All participants with missing } \\
\text { data had the outcome }\end{array}$ & $\begin{array}{l}\text { Assumed that all } \\
\text { had the outcome }\end{array}$ & Denominator included & $\begin{array}{l}\text { Assumed that all } \\
\text { had the outcome }\end{array}$ & Denominator included \\
\hline Worst case scenariot & $\begin{array}{l}\text { Assumed that all had an } \\
\text { unfavourable outcome }\end{array}$ & Denominator included & $\begin{array}{l}\text { Assumed that all had a } \\
\text { favourable outcome }\end{array}$ & Denominator included \\
\hline IMOR 3 & IMOR 3§ & Denominator included & IMOR 1 & Denominator included \\
\hline IMOR 5 & IMOR $5 \S$ & Denominator included & IMOR 1 & Denominator included \\
\hline \multicolumn{5}{|c|}{$\begin{array}{l}\text { IMOR=informative missing odds ratio } \\
\text { *When applying best case scenario, it was ensured it challenges the relative effect by shifting it away from the null value of no effect (see statistical notes } \\
\text { in appendix section } 3 \text { ). } \\
\text { tWhen applying worst case scenario, it was ensured it challenges the relative effect by shifting it closer to the null value of no effect (see statistical notes } \\
\text { in appendix section } 3 \text { ). } \\
\text { fThe "metamiss" command }{ }^{25}{ }^{26} \text { was used to implement the IMOR assumptions in Stata. } \\
\text { §These calculations are applied when the relative effect is less than } 1 \text {. When a relative effect is greater than } 1 \text {, the values for the IMOR are flipped } \\
\text { between the intervention and control arm whereby it is } 1 \text { for the intervention arm. For example, when an original relative effect is greater than } 1 \text {, the } \\
\text { IMOR value for the intervention arm would be } 1 \text { and that of the control arm would be } 5 \text {. }\end{array}$} \\
\hline
\end{tabular}

in relative risk of $25 \%$ for the sensitivity analysis pooled effect estimate (worst case scenario) over the sensitivity analysis pooled effect estimate (complete case analysis) implies that for a relative risk of 0.8 with complete case analysis, the relative risk for the worst case scenario would be 1, and for a relative risk of 1.6 with complete case analysis, the relative risk for the worst case scenario would be 1.2.

\section{Crossing the threshold of the null effect}

For the analysis of the percentage of meta-analyses for which the sensitivity analysis pooled relative effect (assumption) crossed the threshold of the null effect compared with the sensitivity analysis pooled relative effect (complete case analysis), we restricted the sample to the meta-analyses that did not cross the threshold of the null effect under the complete case analysis method.

\section{Changing direction of pooled relative effect}

In the analysis of the percentage of meta-analyses for which the sensitivity analysis pooled relative effect (assumption) changed direction compared with the sensitivity analysis pooled relative effect (complete case analysis), the direction could change from favouring the intervention to favouring the control or vice versa. For this analysis, we restricted the sample to the meta-analyses that crossed the threshold of the null effect under the complete case analysis method.
We reproduced the analyses for outcomes when crossing the threshold of the null effect and when changing direction, comparing the sensitivity analysis pooled relative effect to the original pooled relative effect. In addition, we conducted all the analyses twice: first considering participants with definite missing outcome data, then considering participants with total possible missing outcome data (see table 1). In addition, we explored how heterogeneity varies across the different methods.

\section{Patient and public involvement}

As this project concerns methodology, no patients or public were involved.

\section{Results}

\section{Study characteristics of included meta-analyses}

We previously reported on the details of the 100 eligible systematic reviews ${ }^{16}$ and the 653 randomised controlled trials they considered. ${ }^{8}$ Table 3 summarises the characteristics of the 100 included systematic reviews and the corresponding meta-analyses. Most reported on a drug related outcome $(61 \%)$ and a nonactive control (55\%), assessed a morbidity outcome (56\%), reported an unfavourable outcome (73\%), used the risk ratio (61\%), and applied a fixed effect analysis model (57\%) and Mantel-Haenszel statistical methods $(77 \%)$. The median number of randomised controlled trials per meta-analysis was 6 (interquartile range 3-8). 
Eight meta-analyses included randomised controlled trials that reported no missing data.

\begin{abstract}
Missing data in randomised controlled trials
The results of 400 of the 653 randomised controlled trials $(63 \%)$ mentioned at least one of the predefined categories of participants who might have missing outcome data. Among those 400 trials, the total median percentage of participants with definite missing outcome data was $5.8 \%$ (interquartile range $2.2-14.8 \%) ; 3.8 \%(0-12 \%)$ in the intervention arm and $3.4 \%(0-12 \%)$ in the control arm. The median percentage of participants with potential missing outcome data was $9.7 \%(4.1-19.9 \%)$ and with total possible missing data was $11.7 \%$ (5.6-23.7\%). Only three trials provided a reason for missingness (eg, missing at random).
\end{abstract}

\section{Change in relative effect estimate}

Figure 2 shows the change in the relative effect estimate between the sensitivity analysis pooled effect estimate (assumption) and the sensitivity analysis pooled effect estimate (complete case analysis), when considering participants with definite missing data.

\begin{tabular}{|c|c|}
\hline Characteristics & Estimate \\
\hline Median (interquartile range) No of randomised controlled trials in each meta-analysis & $6(3-8)$ \\
\hline \multicolumn{2}{|l|}{ Type of intervention: } \\
\hline Drug & $61(61.0)$ \\
\hline Surgery or invasive procedure & $24(24.0)$ \\
\hline Other & $15(15.0)$ \\
\hline \multicolumn{2}{|l|}{ Type of control: } \\
\hline Active: drug & $21(21.0)$ \\
\hline Active: surgery or invasive procedure & $18(18.0)$ \\
\hline Non-active: no intervention, standard of care, placebo, or sham & $55(55.0)$ \\
\hline Other & $6(6.0)$ \\
\hline \multicolumn{2}{|l|}{ Outcome category: } \\
\hline Mortality & $21(21.0)$ \\
\hline Morbidity & $56(56.0)$ \\
\hline Patient reported outcomes & $23(23.0)$ \\
\hline \multicolumn{2}{|l|}{ Favourability of outcome*: } \\
\hline Favourable & $27(27.0)$ \\
\hline Unfavourable & $73(73.0)$ \\
\hline Mean (SD) duration of outcome follow-up (months) & $12.5(23.1)$ \\
\hline \multicolumn{2}{|l|}{ Effect measures reported: } \\
\hline Risk ratio & $61(61.0)$ \\
\hline Odds ratio & $39(39.0)$ \\
\hline \multicolumn{2}{|l|}{ Analysis model: } \\
\hline Random effects model & $43(43.0)$ \\
\hline Fixed effect model & $57(57.0)$ \\
\hline \multicolumn{2}{|l|}{ Statistical methods: } \\
\hline Mantel-Haenszel & $77(77.0)$ \\
\hline Inverse variance & $4(4.0)$ \\
\hline Peto & $7(7.0)$ \\
\hline Other & $7(7.0)$ \\
\hline Not reported & $5(5.0)$ \\
\hline \multicolumn{2}{|l|}{ Reported handling method: } \\
\hline Complete case analysis & $2(2.0)$ \\
\hline Assuming no participants with missing data had the event & $3(3.0)$ \\
\hline Assuming all participants with missing data had the event & $2(2.0)$ \\
\hline Not reported & $93(93.0)$ \\
\hline
\end{tabular}

For the four implausible but commonly discussed assumptions, the percentage of meta-analyses with an increased relative effect estimate (shifted away from the null value of 1) was $91 \%$ for the best case scenario assumption, 25\% for the assumption that none of the participants with missing data had the event, and $17 \%$ for the assumption that all participants with missing data had the event. The median increase in the relative effect estimate ranged from $0 \%$ for the worst case scenario assumption to $18.9 \%$ (interquartile range 6.8-38.9\%) for the best case scenario assumption. The percentage of meta-analyses with reduced relative effect estimates (shifted closer towards the null value of 1) was $90 \%$ for the worst case scenario assumption, $38 \%$ for the assumption that none of the participants with missing data had the event, and $75 \%$ for the assumption that all participants with missing data had the event. The median reduction in the relative effect estimate ranged from $0 \%$ for the best case scenario assumption to $30.4 \%$ (interquartile range10.5-77.5\%) for the worst case scenario assumption.

For the plausible assumptions based on the IMOR, the percentage of meta-analyses with an increased relative effect estimate was $85 \%$ for the least stringent assumption (IMOR 1.5) and 88\% for the most stringent assumption (IMOR 5). The median reduction in relative effect estimate ranged from $1.4 \%(0.6-3.9 \%)$ for the IMOR 1.5 assumption to $7.0 \%(2.7-18.2 \%)$ for the IMOR 5 assumption. The appendix presents the details of the percentage change in the relative effect estimate when considering participants with total possible missing data (supplementary results $\mathrm{C}$ ), and stratified by whether the estimate is less than or greater than 1 under the complete case analysis, using either definite missing data or total possible missing data (supplementary results D table 3).

\section{Crossing the threshold of the null effect and change of direction}

Of the 100 meta-analyses, 87 did not cross the threshold of the null effect under the complete case analysis method. Figure 3 shows the number of metaanalyses that crossed the threshold of the null effect when comparing the sensitivity analysis pooled relative effect (assumption) with the sensitivity analysis pooled relative effect (complete case analysis) for each assumption and considering participants with definite missing data. For the four implausible but commonly discussed assumptions, the percentage of meta-analyses that crossed the threshold of the null effect ranged from 1\% (best case scenario and none of the participants with missing data had the event) to $18 \%$ (all participants with missing data had the event) to $60 \%$ (worst case scenario). For the plausible assumptions based on IMOR, the percentage of meta-analyses that crossed the threshold of the null effect ranged from $6 \%$ (least stringent assumption IMOR 1.5 ) to $22 \%$ (most stringent assumption IMOR 5).

The percentage of meta-analyses that changed direction with the two extreme assumptions was 


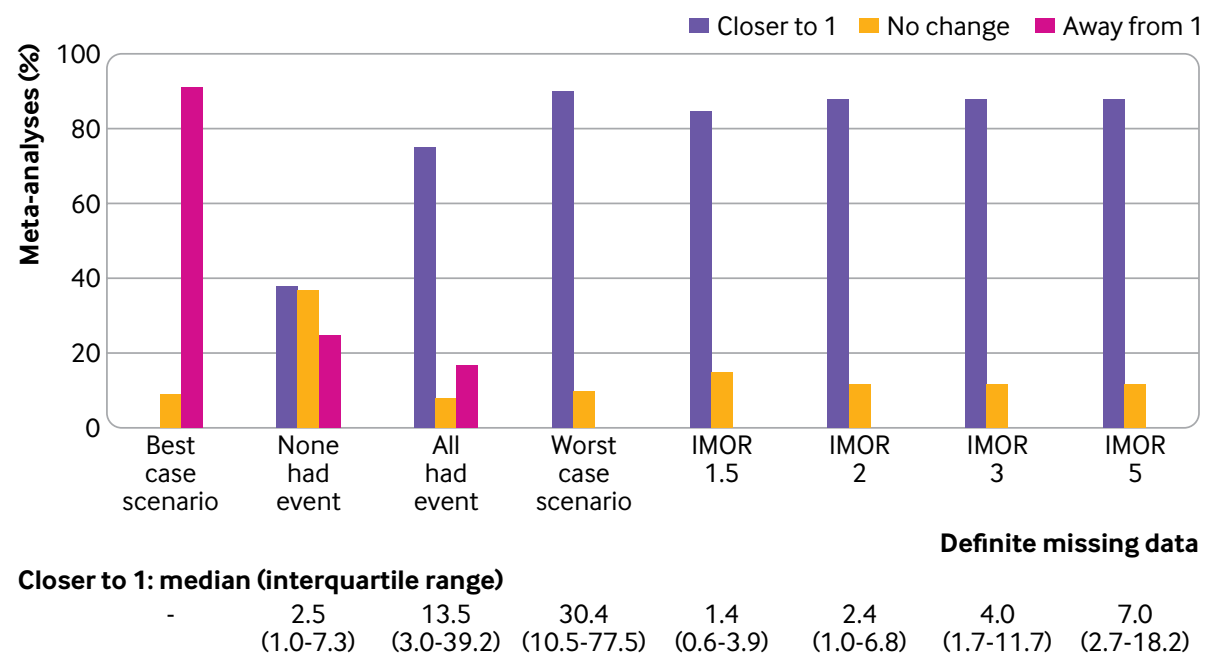

Away from 1: median (interquartile range)

$\begin{array}{ccc}18.9 & 1.6 & 0.9 \\ (6.8-38.9) & (0.8-3.5) & (0.3-2.1\end{array}$

Fig 2 | Change in relative effect estimate (by direction) between the sensitivity analysis pooled effect estimate (assumption) and the sensitivity analysis pooled percentage of meta-analyses effect estimate (complete case analysis) when considering participants with definite missing data. Coloured bars represent the percentage of meta-analyses with change in relative effect estimate (by direction). Numerical values represent the median (interquartile range) for increase and decrease in relative effect estimate $(n=100$, respectively. IMOR=informative missing odds ratio

$26 \%$ for the worst case scenario and $2 \%$ for the most stringent assumption IMOR 5.

The appendix presents the results of meta-analyses that crossed the threshold of the null effect ${ }^{1}$ and changed direction when participants with total possible missing data were considered ${ }^{2}$ (supplementary results A), and when the sensitivity analysis pooled relative effect (assumption) was compared with the original pooled relative effect (supplementary results B).

\section{Change in heterogeneity}

Figure 4 shows the change in heterogeneity across the different methods of handling missing data when considering participants with definite missing data. The median $\mathrm{I}^{2}$ for the original pooled relative effect was $0 \%$ (interquartile range 0-42\%) and for the complete case analysis was $3.6 \%(0-47 \%)$. For the four implausible but commonly discussed assumptions, the median $\mathrm{I}^{2}$ ranged from $0.4 \%$ (none of the participants with missing data had the event) to $20.3 \%$ (all participants with missing data had the event) to $29 \%$ (best case scenario) to $58.6 \%$ (worst case scenario). For the plausible assumptions based on IMOR, the percentage of meta-analyses that crossed the threshold of the null effect ranged from $2.4 \%$ (least stringent assumption IMOR 1.5) to $4.4 \%$ (most stringent assumption IMOR 5).

\section{Discussion}

In the current study, we quantified the change in the effect estimate when applying different methods of handling missing data to the outcomes of participants with definite missing data. When applying plausible assumptions to the outcomes of participants with definite missing data, the median change in relative effect estimate was as high as $7.0 \%$ (interquartile range 2.7-18.2\%). When applying implausible but commonly discussed assumptions, the median change in the relative effect estimate was as large as $30.4 \%$ (10.5-77.5\%).

We also examined how different methods of handling missing data alter crossing the threshold of the null effect of pooled effect estimates of dichotomous outcomes. Even when applying plausible assumptions to the outcomes of participants with definite missing data, almost a quarter (22\%) of meta-analyses crossed the threshold of the null effect. When applying implausible but commonly discussed assumptions, the percentage of systematic reviews that crossed the threshold of the null effect was as high as $60 \%$ with the worst case scenario.

\section{Strengths and limitations of this study}

In this study we assessed the effect of using different assumptions (both commonly discussed and more plausible) on a large number of published metaanalyses of pairwise comparisons targeting a wide range of clinical areas. Strengths of our study include a detailed approach to assessing participants with missing data, and accounting for participants with potential missing data in our analyses. We used two statistical approaches to assess the risk of bias associated with missing outcomes: change in effect estimate and crossing the threshold of the null effect. Although the approach of crossing the threshold of the null effect has been criticised as the basis for decision making, ${ }^{28}$ we used it to assess the robustness of the meta-analysis effect estimate (ie, when conducting sensitivity meta-analyses using different methods of handling missing data). We are confident that if results 


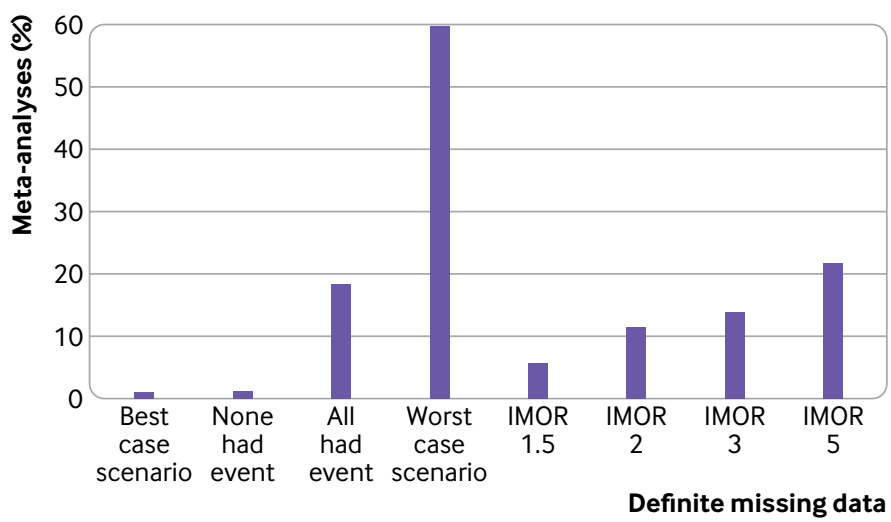

Fig 3 | Results of meta-analyses that crossed the threshold of null effect when considering participants with definite missing data and comparing the sensitivity analysis pooled relative effect (assumption) with the sensitivity analysis pooled relative effect (complete case analysis) ( $n=87$ systematic reviews that did not cross the threshold of null effect under the complete case analysis method). IMOR=informative missing odds ratio
2020 in the chronological order of their publication and selected the first 15 that met our eligibility criteria. Then we applied the same methodology stated in the methods section to assess the reporting and handling of missing data in the 15 eligible meta-analyses. As with the 2012 sample of systematic reviews reported on here, most of the systematic reviews published in 2020 did not explicitly plan (in the Methods section) to consider any category of missing data $(60 \%)$, did not report (in the Results section) data for any category of missing data (67\%), did not report the proportion of missing data for each trial and for each arm (67\%), did not explicitly state a specific analytical method for handling missing data (80\%), and did not provide a justification for the analytical method used to handle missing data (100\%).

Another limitation was our focus on systematic reviews with statistically significant results. Although this prevented us from assessing the change in effect estimate for meta-analyses with non-statistically significant results, it allowed us to focus on reviews that are more likely to influence clinical practice.

We acknowledge the possible superiority of using the random IMOR over fixed IMOR. However, the variance or uncertainty increases as a function of the proportion of missingness and the variance of the IMOR parameter. ${ }^{125}$ Hence, uncertainty increases even with fixed IMORs although to a smaller extent. Fully introducing uncertainty to IMORs by using random IMOR (although it was not feasible for this study) would exaggerate our results. Specifically, we would expect a further increase in the proportion of meta-analyses in which the confidence interval would cross the line of no effect and thus lose statistical significance, larger within study variance, and further downgrading of the certainty of the evidence. Hence, the switch to random IMOR would only reinforce the inferences from our work that we already make.

\section{Interpretation of findings}

Experience and acceptance for using plausible assumptions is growing as a result of face validity. ${ }^{1134}$ The advantage of the IMOR approach is that it

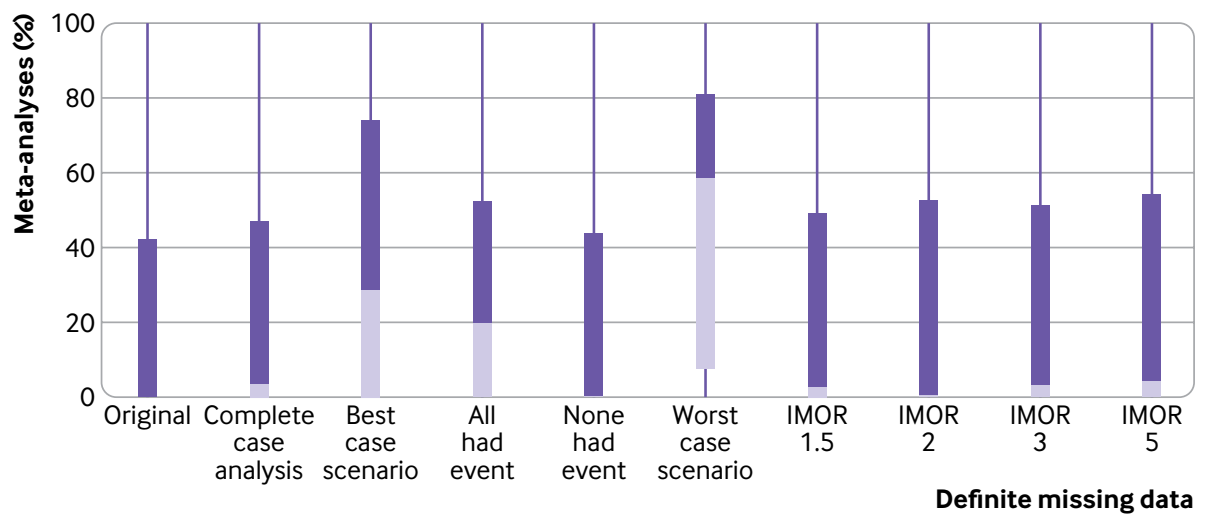

Fig 4 | Change in heterogeneity $\left(\mathrm{I}^{2}\right)$ across different methods of handling missing data. IMOR=informative missing odds ratio 
provides a tool for review authors to challenge the robustness of effect estimates by applying increasingly stringent assumptions. ${ }^{25}$ This approach allows the review authors to choose the IMOR value or range of values based on their clinical judgment or expert opinion. Only if the effect estimate is robust to the most conservative and plausible scenario can it be concluded that the evidence is at low risk of bias from missing data without additional sensitivity analyses. The selection of the assumptions about missing data should be determined a priori with researchers blinded to the extent of missing data in the included trials to avoid data dredging. Thus we should rate down for missing data when it might change inferences. Inferences could be that treatment is of benefit (versus not of benefit) or that treatment achieves an important benefit. That would require a threshold of minimal clinically important difference and a check to see if the threshold was crossed initially and then after accounting for minimal clinically important difference. We could not do that unless we knew the minimal clinically important difference.

Almost a quarter (22\%) of meta-analyses crossed the threshold of the null effect when we used a conservative approach to test robustness (ie, applying plausible assumptions to the outcomes of participants with definite missing data). When using the same conservative approach, up to a quarter of metaanalyses had a change of at least $18 \%$ in their relative effect estimates (based on the $75^{\text {th }}$ centile for IMOR 5 , see median and interquartile range of IMOR 5 in fig 2). These findings mean that a substantive percentage of meta-analyses is at serious risk of bias associated with missing outcome data. Findings such as these should lead systematic review authors to rate down the certainty of evidence for risk of bias. Our results highlight the importance of minimising missing data for clinical trials ${ }^{3536}$ by better reporting and handling of missing data. ${ }^{81637}$

With the two assumptions that all participants with missing data had the event and none of the participants with missing data had the event, the size and direction of the confidence intervals are unpredictable; sometimes the effect estimate is shifted closer to 1 and sometimes away from 1 . Thus, these assumptions are not helpful for challenging the robustness of the effect estimate. By design, the effect estimate using the best case scenario assumption is shifted in the opposite direction of challenging the robustness and should not be used for that purpose. The worst case scenario consistently challenges the robustness of the effect estimate as it is shifted towards the null effect, but the implausibility of its underlying assumptions makes it a poor choice for sensitivity analyses. If the effect estimate is robust to the worst case scenario it can be concluded that the evidence is at low risk of bias from missing data, without proceeding with further sensitivity analyses.

As for the change in heterogeneity, since with the implausible but common assumptions, the size and direction of the confidence intervals are unpredictable, consequently the change in $\mathrm{I}^{2}$ was observed to be unpredictable. As for the plausible assumptions, since uncertainty is taken into account, confidence intervals tend to be wider and more overlapping, leading to low $\mathrm{I}^{2}$ values. Future studies might need to study whether a certain change in $\mathrm{I}^{2}$ in a sensitivity analysis is worth rating down the certainty of evidence as a result of inconsistency.

\section{Conclusion and implications}

The findings of this study show the potential impact of missing data on the results of systematic reviews. This has implications when both the risk of bias associated with missing outcome data is assessed and the extent of missing outcome data in clinical trials needs to be reduced.

Systematic review authors should present the potential impact of missing outcome data on their effect estimates and, when these suggest lack of robustness of the results, rate down the certainty of evidence for risk of bias. For practical purposes, authors of systematic reviews might wish to use statistical software that allows running assumptions about missing data (eg, Stata). As for users of the medical literature, a rule of thumb on how to judge risk of bias associated with missing outcome data at the trial level is needed. This would account for factors such as percentage of missing data in each study arm, the ratio of missing data to event rate for each study arm (ie, the higher the ratio, the larger the change), fragility of statistical significance (ie, borderline significance), the magnitude of the effect estimate (ie, the larger the effect estimate, the smaller the change), and the duration of follow-up (ie, the longer the duration of follow-up, the higher the percentage of missing data).

We acknowledge that assessing the impact of missing data with crossing the threshold of the null effect might be insensitive. Thus, when using this approach and the threshold of null effect is crossed, then rate down the certainty for risk of bias associated with missing data. If threshold of null effect is not crossed, it might be valuable to then evaluate the change in effect estimate to assess whether the relative effect goes from an important to an unimportant effect. If the latter happens, then rate down the certainty for risk of bias associated with missing data. However, the judgment of whether the change in effect estimate is clinically significant requires using minimal clinically important difference, which varies by clinical question. Thus, it would be ideal to reproduce this study in specific subjects of medical science with clearly defined minimal clinically important differences and using random IMOR instead of fixed IMOR.

Future research could also validate some of the findings of this study. For example, this study could be reproduced using individual participant data metaanalyses and findings compared with the current study. In addition, individual participant data meta-analyses would allow testing other imputation methods, such as multiple imputations. 


\section{AUTHOR AFFILIATIONS}

${ }^{1}$ Department of Internal Medicine, American University of Beirut Medical Center, PO Box 11-0236, Riad-El-Solh Beirut 1107 2020, Beirut, Lebanon

${ }^{2}$ Cochrane Netherlands and Julius Center for Health Sciences and Primary Care, University Medical Center Utrecht, Utrecht University, Utrecht, Netherlands

${ }^{3}$ Wolfson Palliative Care Research Centre, Hull York Medical School, University of Hull, Hull, UK

${ }^{4}$ Department of Health Research Methods, Evidence, and Impact, McMaster University, Hamilton, ON, Canada

${ }^{5}$ Pharmaceutical Sciences Post Graduate Course, University of Sorocaba, UNISO, Sorocaba, Sao Paulo, Brazil

${ }^{6}$ Department of Medicine, University of Toronto, Toronto, ON, Canada

${ }^{7}$ Chinese Evidence-based Medicine Center, West China Hospital, Sichuan University, Chengdu, China

${ }^{8}$ Departments of Medicine and Biomedical and Health Informatics, University of Missouri-Kansas City, Kansas City, MO, USA

${ }^{9}$ Department of Evaluative Clinical Sciences, Sunnybrook Health Sciences Centre, Toronto, ON, Canada

${ }^{10}$ Department of Epidemiology, Harvard TH Chan School of Public Health, Boston, MA, USA

${ }^{11}$ Department of Anaesthesia, McMaster University, Hamilton, ON, Canada

${ }^{12}$ The Michael G DeGroote Institute for Pain Research and Care, McMaster University, Hamilton, ON, Canada

${ }^{13}$ Canadian Veterans Chronic Pain Centre of Excellence, Hamilton, ON, Canada

${ }^{14}$ Department of Medicine, McMaster University, Hamilton, ON, Canada

Contributors: LAK, AMK, GG, HJS, and EAA conceived and designed the paper. $L A K$ and BD developed the full text screening form. LAK developed the data abstraction form. LAK, BD, YC, LCL, AA, LL, RM, SK, RW, JWB, and AD abstracted the data. LAK and AMK analysed the data. LAK, LH, RJPMS, and EAA interpreted the results. LAK, AMK, LH, RJPMS, and EAA drafted the manuscript. All authors revised and approved the final manuscript. EAA is the lead author and guarantor. LAK attests that all listed authors meet authorship criteria and that no others meeting the criteria have been omitted.

Funding: This study was funded by the Cochrane Methods Innovation Fund. The funder had no role in considering the study design or in the collection, analysis, interpretation of data, writing of the report, or decision to submit the article for publication.

Competing interest: All authors have completed the ICMJE uniform disclosure form at www.icmje.org/coi_disclosure.pdf and declare: support from the Cochrane Methods Innovation Fund for the submitted work, no financial relationships with any organisations that might have an interest in the submitted work in the previous three years; no other relationships or activities that could appear to have influenced the submitted work.

Ethical approval: Not required.

Data sharing: Data are available on reasonable request from the corresponding author at ea32@aub.edu.lb. Proposals requesting data access will need to specify how it is planned to use the data.

The lead author EAA (the manuscript's guarantor) affirms that the manuscript is an honest, accurate, and transparent account of the study being reported; that no important aspects of the study have been omitted; and that any discrepancies from the study as planned (and, if relevant, registered) have been explained.

Dissemination to participants and related patient and public communities: Lay information on the key results of the study will be made available on request from the corresponding author.

This manuscript has been deposited as a preprint.

This is an Open Access article distributed in accordance with the Creative Commons Attribution Non Commercial (CC BY-NC 4.0) license, which permits others to distribute, remix, adapt, build upon this work non-commercially, and license their derivative works on different terms, provided the original work is properly cited and the use is noncommercial. See: http://creativecommons.org/licenses/by-nc/4.0/.

1 National Research Council. The Prevention and Treatment of Missing Data in Clinical Trials. The National Academic Press, 2010.

2 Akl EA, Briel M, You JJ, et al. Potential impact on estimated treatment effects of information lost to follow-up in randomised controlled trials
(LOST-IT): systematic review. BMJ 2012;344:e2809. doi:10.1136/ bmi.e2809

3 Bell ML, Fiero M, Horton NJ, Hsu CH. Handling missing data in RCTs; a review of the top medical journals. BMC Med Res Methodol 2014:14:118. doi:10.1186/1471-2288-14-118

4 Fielding S, Ogbuagu A, Sivasubramaniam S, MacLennan G, Ramsay CR. Reporting and dealing with missing quality of life data in RCTs: has the picture changed in the last decade?Qual Life Res 2016:25:2977-83. doi:10.1007/s11136-016-1411-6

5 Hussain JA, Bland M, Langan D, Johnson MJ, Currow DC, White IR. Quality of missing data reporting and handling in palliative care trials demonstrates that further development of the CONSORT statement is required: a systematic review. / Clin Epidemiol 2017;88:81-91. doi:10.1016/j.jclinepi.2017.05.009

6 Powney M, Williamson P, Kirkham J, Kolamunnage-Dona R. A review of the handling of missing longitudinal outcome data in clinical trials. Trials 2014;15:237. doi:10.1186/1745-6215-15-237

7 Ibrahim F, Tom BD, Scott DL, Prevost AT. A systematic review of randomised controlled trials in rheumatoid arthritis: the reporting and handling of missing data in composite outcomes. Trials 2016;17:272. doi:10.1186/s13063-016-1402-5

8 Kahale LA, Diab B, Khamis AM, et al. Potentially missing data are considerably more frequent than definitely missing data: a methodological survey of 638 randomized controlled trials. J Clin Epidemiol 2019;106:18-31.

9 Hallgren KA, Witkiewitz K, Kranzler HR, et al, In conjunction with the Alcohol Clinical Trials Initiative (ACTIVE) Workgroup. Missing data in alcohol clinical trials with binary outcomes. Alcohol Clin Exp Res 2016;40:1548-57. doi:10.1111/acer.13106

10 Guyatt GH, Ebrahim S, Alonso-Coello P, et al. GRADE guidelines 17: assessing the risk of bias associated with missing participant outcome data in a body of evidence. J Clin Epidemiol 2017;87:1422. doi:10.1016/j.jclinepi.2017.05.005

11 Spineli LM. An empirical comparison of Bayesian modelling strategies for missing binary outcome data in network meta-analysis. BMC Med Res Methodol 2019;19:86. doi:10.1186/s12874-019-0731-y

12 Akl EA, Kahale LA, Ebrahim S, Alonso-Coello P, Schünemann HJ, Guyatt GH. Three challenges described for identifying participants with missing data in trials reports, and potential solutions suggested to systematic reviewers. J Clin Epidemiol 2016;76:147-54. doi:10.1016/j.jclinepi.2016.02.022

13 Kahale LA, Guyatt GH, Agoritsas T, et al. A guidance was developed to identify participants with missing outcome data in randomized controlled trials. J Clin Epidemiol 2019;115:55-63. doi:10.1016/j. jclinepi.2019.07.003

14 Cochrane Handbook for Systematic Reviews of Interventions Version 5.1.0 [updated March 2011]. The Cochrane Collaboration, 2011. www.handbook.cochrane.org. 2011.

15 Akl EA, Kahale LA, Agarwal A, et al. Impact of missing participant dat for dichotomous outcomes on pooled effect estimates in systematic reviews: a protocol for a methodological study. Syst Rev 2014;3:137. doi:10.1186/2046-4053-3-137

16 Kahale LADB, Diab B, Brignardello-Petersen R, et al. Systematic reviews do not adequately report or address missing outcome data in their analyses: a methodological survey. J Clin Epidemiol 2018:99:14-23. doi:10.1016/j.jclinepi.2018.02.016

17 Harris PA, Taylor R, Thielke R, Payne J, Gonzalez N, Conde JG. Research electronic data capture (REDCap)--a metadata-driven methodology and workflow process for providing translational research informatics support. J Biomed Inform 2009;42:377-81. doi:10.1016/j. jbi.2008.08.010

18 SPSS. IBM SPSS Statistics for Windows. Version 21.0. IBM Corp, 2013.

19 StataCorp. Stata Statistical Software: Release 12. In: Station C, ed. StataCorp LP, 2011.

20 AkI EA, Kahale LA, Agoritsas T, et al. Handling trial participants with missing outcome data when conducting a meta-analysis: a systematic survey of proposed approaches. Syst Rev 2015;4:98 doi:10.1186/s13643-015-0083-6

21 White IR, Welton NJ, Wood AM, Ades AE, Higgins JP. Allowing for uncertainty due to missing data in meta-analysis--part 2: hierarchical models. Stat Med 2008;27:728-45. doi:10.1002/sim.3007

22 White IR, Higgins JP, Wood AM. Allowing for uncertainty due to missing data in meta-analysis--part 1: two-stage methods. Stat Med 2008;27:711-27. doi:10.1002/sim.3008

23 Geng EH, Bangsberg DR, Musinguzi N, et al. Understanding reasons for and outcomes of patients lost to follow-up in antiretroviral therapy programs in Africa through a sampling-based approach. J Acquir Immune Defic Syndr 2010;53:405-11. doi:10.1097/ QAl.0b013e3181b843fo

24 Geng EH, Emenyonu N, Bwana MB, Glidden DV, Martin JN. Samplingbased approach to determining outcomes of patients lost to follow-up in antiretroviral therapy scale-up programs in Africa. IAMA 2008:300:506-7. doi:10.1001/jama.300.5.506

25 White IR, Higgins JP. Meta-analysis with missing data. Stata / 2009;9:57-69. doi:10.1177/1536867X0900900104 
26 Chaimani A, Mavridis D, Higgins JPT, Salanti G, White IR. Allowing for informative missingness in aggregate data meta-analysis with continuous or binary outcomes: Extensions to metamiss. Stata J 2018;18:716-40. doi:10.1177/1536867X1801800310

27 Higgins JP, White IR, Wood AM. Imputation methods for missing outcome data in meta-analysis of clinical trials. Clin Trials 2008;5:225-39. doi:10.1177/1740774508091600

28 Wasserstein RL, Schirm AL, Lazar NA. Moving to a World Beyond " $p$ s 0.05 " Taylor \& Francis, 2019.

29 Ebrahim S, Akl EA, Mustafa RA, et al. Addressing continuous data for participants excluded from trial analysis: a guide for systematic reviewers. / Clin Epidemiol 2013:66:1014-1021.e1.

30 Ebrahim S, Johnston BC, Akl EA, et al. Addressing continuous data measured with different instruments for participants excluded from trial analysis: a guide for systematic reviewers. J Clin Epidemiol 2014;67:560-70. doi:10.1016/j.jclinepi.2013.11.014

31 Sullivan TR, Yelland LN, Lee KJ, Ryan P, Salter AB. Treatment of missing data in follow-up studies of randomised controlled trials: A systematic review of the literature. Clin Trials 2017;14:387-95. doi:10.1177/1740774517703319

32 Babic A, Tokalic R, Amílcar Silva Cunha J, et al. Assessments of attrition bias in Cochrane systematic reviews are highly inconsistent and thus hindering trial comparability. BMC Med Res Methodol 2019;19:76. doi:10.1186/s12874-019-0717-9

33 Shivasabesan G, Mitra B, O’Reilly GM. Missing data in trauma registries: A systematic review. Injury 2018;49:1641-7. doi:10.1016/j.injury.2018.03.035

34 Mavridis D, White IR. Dealing with missing outcome data in metaanalysis. Res Synth Methods 2020;11:2-13.

35 Hollestein LM, Carpenter JR. Missing data in clinical research: an integrated approach. BrJ Dermatol 2017;177:1463-5. doi:10.1111/ bjd. 16010

36 Little RJ, Cohen ML, Dickersin K, et al. The design and conduct of clinical trials to limit missing data. Stat Med 2012;31:3433-43. doi:10.1002/sim.5519

37 Akl EA, Shawwa K, Kahale LA, et al. Reporting missing participant data in randomised trials: systematic survey of the methodological literature and a proposed guide. BMJ Open 2015;5:e008431. doi:10.1136/bmjopen-2015-008431

Supplementary information: additional tables, statistical notes, and results 